\title{
Revolutionizing Optical Fiber Transmission and Networking Using the Orbital Angular Momentum of Light
}

\author{
Antonella Bogoni \\ Scuola Superiore Sant'Anna \\ Pisa, Italy \\ antonella.bogoni@sssup.it \\ Mirco Scaffardi \\ CNIT \\ Pisa, Italy \\ Marc Sorel \\ University of Glasgow \\ Glasgow, United Kingdom \\ Bert Offrein \\ IBM \\ Zurich, Switzerland
}

\author{
Yabin Ye, Gernot Göger \\ Huawei Technologies Duesseldorf GmbH \\ Munich, Germany
}

Sophie Larochelle

Université Laval

Québec City, Canada

Siyuan $\mathrm{Yu}$

University of Bristol

Bristol, United Kingdom

Piotr Rydlichowski

Poznań Supercomputing and Networking Centre

Poznań, Poland

\begin{abstract}
The ROAM (Revolutionizing optical fiber transmission and networking using the Orbital Angular Momentum of light) project investigates the orbital angular momentum (OAM) modes of light for communications and networking applications. OAM modes are exploited as a disruptive means of increasing fibre transmission capacity and as switching resource to improve the switches scalability and power consumption in data-centre scenarios.
\end{abstract}

Keywords-orbital angular momentum, optical multiplexing, optical networking, data center.

\section{INTRODUCTION}

Current optical transmission technologies are facing some major and fundamental challenges in that further increase in single mode fibre (SMF) transmission capacity is being limited by two main physical factors, namely optical bandwidth and nonlinearity [1]. Laboratory systems have demonstrated near full use of the $11.4 \mathrm{THz}$ optical bandwidth available in $\mathrm{C}+\mathrm{L}$ bands, achieving high capacity by a combination of dense frequency domain multiplexing, high order modulation formats and coherent detection [2]. Further increase of the optical bandwidth by means of additional frequency-domain multiplexing channel count is limited by nonlinear effects. The last option is to explore spatial or mode division multiplexing schemes [3], [4]. Further increase in core count is likely to increase inter-core coupling. To fully use the cross-section of the fibre, mode division multiplexing or few-mode communications schemes have been explored. Inter-modal coupling presents a major technical challenge, and MDM schemes require significant multi-input and multi-output (MIMO) processing power that limits the scalability of such schemes [5].

The transmission of multiplexed OAM channels in the free space has been recently demonstrated at $400 \mathrm{~Gb} / \mathrm{s}$ with $4 \mathrm{OAM}$ modes up to $120 \mathrm{~m} \mathrm{[6]} \mathrm{and} \mathrm{at} 8.16 \mathrm{~Tb} / \mathrm{s}$ with 52 modes for few meters [7]. To make transmission of OAM light practical for longer distances, special optical fibres supporting OAM modes are necessary [8]. In this view, the transmission of 9 OAM modes through an hollow-core fibre has been demonstrated [9].

Concerning the generation of OAM modes, these can be impressed to a Gaussian beam by means of passive devices as spiral phase plates [10] or active devices as spatial light modulators (SLMs) [4]. Recently, research and development of integrated devices capable of generating OAM modes have been undertaken. Microrings [11], circular grating couplers cascaded to star couplers [12] and hybrid 3D integrated circuits 
[13] have been demonstrated to be compact sources to generate light carrying OAM.

Against such backgrounds, ROAM (Revolutionizing optical fiber transmission and networking using the Orbital Angular Momentum of ligh) project proposes to exploit the use of OAM modes in optical fibres to form a unique type of mode division multiplexing scheme - OAM multiplexing. The aim is to circumvent some of the fundamental limitations faced by other mode division schemes.

For what concerns the switching scenario, nowadays the commercial switches employed in the data-centres are electronic switches [14]. Due to the continuous increasing dimension of the data centres [15] the number of Ethernet switches necessary to connect the servers is rising

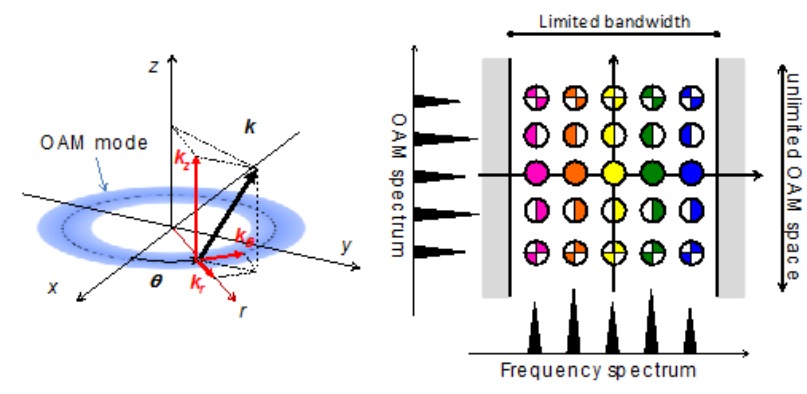

Figure 1. Left: schematic representation of the cylindrical optical mode. Right: central concept of the project proposal.

tremendously, thus the scalability of current electrical interconnection networks will be soon limited by power consumption and power dissipation and footprint issues [16]. Due to the aforementioned issues optical switching is gaining interest [17]. It allows to exploit multiple switching domains, thus enhancing the switches scalability. The traditional switching domains for a flexible switching of data packets are space, wavelength, and time [18].

Within this network scenario, we propose for the first time to exploit OAM domain as an additional switching domain within a multi-layer interconnection network to substantially improve the scalability in data centre applications and overcome at the same time power consumption issues.

\section{CONCEPT}

\section{A. Overall concept}

The OAM of photons is associated with the helical phase front of optical modes, with the Poynting vector following a spiral trajectory. Fig. 1 (left) is a classical picture of the cylindrical optical mode, where the components $\mathrm{k}_{\mathrm{z}}, \mathrm{k}_{\mathrm{r}}$ and $\mathrm{k}_{\theta}$ of the wave vector $\mathrm{k}$ are quantised by the boundary conditions, giving rise to mode indices $[\mathrm{m}, \mathrm{l}] . \mathrm{m}$ is the number of field modes in the radial direction while 1 is the quantum number of azimuthal phase eigenfunction $\Phi=\exp (\mathrm{j} 1 \theta)$. The orthogonal eigen-mode set supported by a fibre is characterised by a unique set of $[\mathrm{m}, 1]$, corresponding to sets of $\mathrm{k}_{\mathrm{r}}$ and $\mathrm{k}_{\theta}$. 1 represents the quantization of the orbital angular momentum of the photons in the optical modes and can take positive, 0 , or

This work has been supported by the EC through H2020 project ROAM (grant no: 645361, www.roam-project.eu). negative integer values. The central concept of this proposal is illustrated by the diagram in Fig. 1 (right). An additional dimension of multiplexing is made available by expanding each carrier frequency/wavelength into multiplex OAM channels, thus increasing the spectral efficiency. This is allowed by the orthogonality between OAM modes. Moreover a new dimension of resources for networking, in addition to wavelength, time and space, has been made available.

\section{B. OAM-based multiplexing enabling high-capacity transmission in optical fibre}

In OAM multiplexing schemes, modes with only one variable index 1 are used. This is achieved by designing and fabricating OAM fibres that support modes with $\mathrm{m}=1$ (namely, singlemode in the radial direction). This results in a one-dimensional mode space, which simplifies the OAM multiplexing scheme very significantly compared to few-mode schemes. It is straightforward to map communications channels to OAM modes on a one-to-one basis. This makes multiplexing and demultiplexing a passive optical process [19]. The coupling between the OAM modes happens mostly between adjacent modes, which results in a near-diagonal MIMO matrix with only significant values in the terms just above or below diagonal, decaying away quickly. Signal processing power can potentially sustain OAM multiplexing up to 100 channels. The mode area of all OAM modes in a fibre scales approximately linearly with the number of supported OAM. Therefore, the increase in channel count does not increase the total optical signal power density, thus reducing the nonlinearity effect due to increased channel count. As the channel mapping processes are passive optical mode conversion processes, in principle they do not impact on the optical signal format usable. OAM multiplexing is compatible to all existing transmission technologies, including overlaying onto WDM multiplexing. In terms of fibre technology, OAM fibre maintains cylindrical symmetry, which means that they can be manufactured using existing SMF technology. Better scalability results from several factors. Firstly it is possible to use sparse mapping, where not all OAM modes are used. Furthermore it is possible to use optical equalization techniques to suppress linear crosstalk between the channels.

\section{OAM as networking resource enabling high-scalable and power-efficient switches for data-centres}

The basic concept of switching exploiting the OAM-based interconnection network has as cornerstone the optical multilayer architecture based on OAM and wavelength domain. $\mathrm{N}$ independent line cards, each one with $\mathrm{M}$ input/output ports, are interconnected. The switching among the ports is done in the wavelength domain, i.e. the destination port is determined by the wavelength of the transmitted signal, while the switching among the cards is implemented with an OAMbased switch. In previous research works the destination card was selected by driving a space switching matrix, which had the disadvantage of high insertion losses. We propose to address the signal to a destination card by assigning a proper OAM mode to the transmitted signal. The assigned OAM mode determines univocally the destination card, independently of the signal wavelength. 
The basic concept of switching exploiting the OAM-based interconnection network is illustrated in Fig. 2.

\section{TECHNOLOGY}

For fabrication of fibers supporting OAM modes, numerical methods to find parameters maximizing the number of OAM modes and their separation are used, subject to fabrication constraints. Solving for modes in multi-step-index fibres having a more complex profile than a single core and cladding can be done using the transfer matrix method. The optimisation procedure considers fabrication constraints. In general, higher index contrast leads to higher attenuation. An air-core (hollow tube fibre) dramatically increases the index contrast, however, surface roughness of the air/SiO2 interface causes increased losses. Another design approach for OAM fibre is to use an inverse parabolic graded index profile

\section{TECHNOLOGICAL IMPACT}

The ROAM project main goal will be to develop and demonstrate OAM-based fibre communication and networking. A 10x improvement of capacity in fibre communication, and a $10 \mathrm{x}$ improvement in scalability and power consumption in switching for data-centres applications are the expected outcomes, which will have a significant industrial impact for both data-centre infrastructure manufacturing and data-centre service providers.

Moreover, the technical solutions developed within the ROAM project will have a substantial environmental impacts, through the development of green (low-power) integrated technological solutions, and societal impacts by contributing to the transformation of communication network infrastructures.

Besides the system advancements and their direct benefits

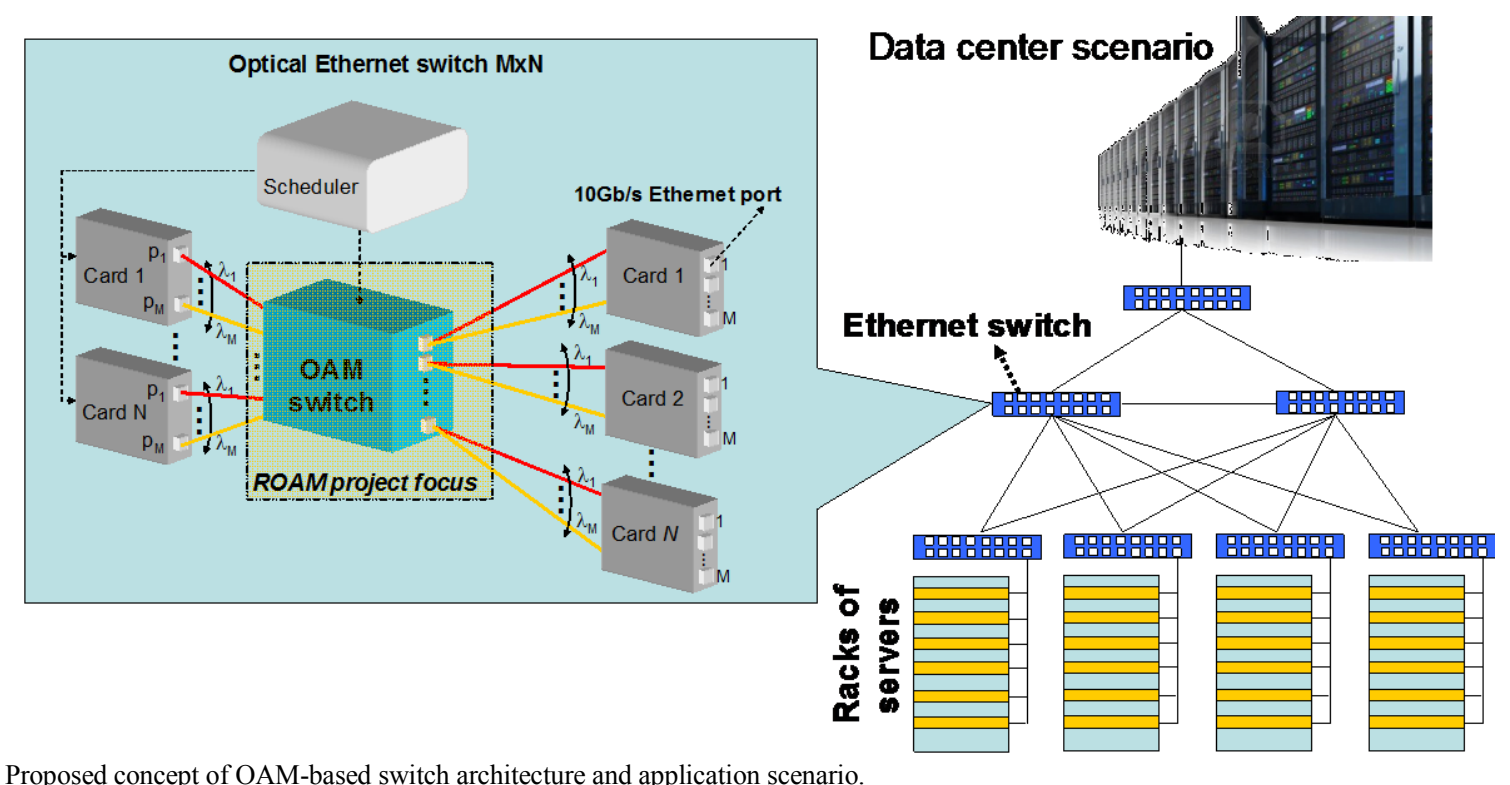

Figure 2. Proposed concept of OAM-based switch architecture and application scenario.

(IPGIF). IPGIF fibres are expected to be more robust to fibre bends, easier to couple with a free-space beam, less sensitive to manufacturing imperfections.

For OAM generation and detection, our approach is based on the smallest integrated OAM components reported so far [20]. The devices use an azimuthal excitation scheme using the combination of whispering gallery mode and angular grating (WGM-AG) fabricated on silicon-on-insulator wafers. This configuration has several advantages, very fast tuning of the OAM quantum number, emitting or receiving of multiple coaxial OAM modes with precise control of the OAM spectrum, very compact devices $(10 \mu \mathrm{m}$ in diameter [20]), optoelectronic integration. Very large arrays of switches and transceivers can be fabricated with standard CMOS technologies thereby providing scalability and flexibility of the components. Silicon will enable the fabrication of compact modules that integrate OAM transceivers with fast optical modulators, detectors, laser sources, and all the control electronics required to drive the components and process the information. to data centres and communication systems, the basic technology developed by ROAM will provide a much broader impact.

The much reduced footprint of this technology together with its potential for integrating large arrays and matrices of OAM emitters on the same silicon chip is likely to be of great interest to several other applications.

For example, OAM beams have a great potential for direct optical trapping and transport of micron sized particles, both inorganic and biological. Conventional optical tweezers use an external laser and mode conversion optics, coupled via freespace to the microscope system. One of the opportunities for the devices being developed by ROAM is to replace these external systems with waveguide devices as integral parts of the sample analysis and processing cell that can trap, mix, sort, and transport microscopic particles. Ultimately, the integration of complex matrices of OAM emitters with conventional labon-a-chip technologies can provide a compact and highly functional approach for the development of a new class of integrated devices. 
The ROAM technology would also provide an extremely appealing approach for quantum information processing. In fact, optical technologies are considered one of the most promising approaches to realizing a truly scalable quantum technology, where quantum information can be encoded and manipulated in any of the many degrees of freedom of the photon. OAM is one such degree of freedom, and has been used to demonstrate a broad range of free-space quantum optics experiments, including the generation of OAM entangled states, high-dimensional quantum key distribution with enhanced security, higher data rates and increased noise resilience, high-dimensional entanglement, and entanglement with very high angular. All implementations of OAM-based quantum optics experiments to-date have used discrete and bulk optical elements. Exploitation of the OAM degree of freedom with integrated waveguide circuits would bring significant advances and enable the development of novel and powerful quantum information systems that harness the scalability of precision of integrated quantum photonics.

The packaging activity carried on in the project can have significant impact on the development of devices to be employed for industrial applications.

\section{REFERENCES}

[1] A. Ellis, J. Zhao, D. Cotter, "Approaching the non-linear Shannon limit" J. Lightw. Technol., vol. 28, no.4, pp. 423-433, 2010.

[2] A. Sano, H. Masuda, T. Kobayashi, M. Fujiwara, K. Horikoshi, E. Yoshida, Y. Miyamoto, M. Matsui, M. Mizoguchi, H. Yamazaki, Y. Sakamaki, and H. Ishii, "69.1-Tb/s (432 x 171-Gb/s) C- and Extended LBand Transmission over $240 \mathrm{~km}$ Using PDM-16-QAM Modulation and Digital Coherent Detection,' OFC'2010, San Diego, CA,

[3] H. Takara, A. Sano, T. Kobayashi, H. Kubota, H. Kawakami, A. Matsuura, Y.Miyamoto, Y. Abe, H. Ono, K. Shikama, Y. Goto, K. Tsujikawa, Y. Sasaki, I. Ishida, K. Takenaga, S. Matsuo, K. Saitoh, M. Koshiba, and T. Morioka, "1.01-Pb/s (12 SDM/222 WDM/456 Gb/s) Crosstalk-managed Transmission with $91.4-\mathrm{b} / \mathrm{s} / \mathrm{Hz}$ Aggregate Spectral Efficiency," presented at the ECOC'2012, Amsterdam, The Netherlands, Jun. 2012.

[4] J. Wang et al., "Terabit free-space data transmission employing orbital angular momentum multiplexing," Nature Photonics, Vol. 6, p. 488 (2012).

[5] V.A.J.M. Sleiffer, Y. Jung, V. Veljanovski, R.G.H. van Uden, M. Kuschnerov, H. Chen, B. Inan, L. Grüner Nielsen, Y. Sun, D.J.
Richardson, S.U. Alam, F. Poletti, J.K. Sahu, A. Dhar, A.M.J. Koonen, B. Corbett, R. Winfield, A.D. Ellis, and H. de Waardt, "73.7 Tb/s (96 x $3 \times 256 \mathrm{~Gb} / \mathrm{s}$ ) mode division multiplexed DP16QAM transmission with inline MMEDFA," presented at the ECOC'2012, Amsterdam, The Netherlands, Jun. 2012.

[6] R. Yongxiong et al., "Experimental characterization of a $400 \mathrm{Gbit} / \mathrm{s}$ orbital angular momentum multiplexed free-space optical link over 120 m", Optics Letters, Vol. 41, n. 3, p. 622 (2016).

[7] J. Wang et al., "Ultra-High 435-bit/s/Hz Spectral Efficiency Using NDimentional Multiplexing and Modulation Link with Pol-Muxed 52 Orbital Angular Momentum (OAM) Modes Carrying Nyquist 32-QAM Signals", Proc. ECOC, Valencia (2015).

[8] B. Ung et al., "Few-mode fiber with inverse-parabolic graded index profile for transmission of OAM-carrying modes", Optics Express, Vol. 22, n. 15, p. 18044 (2014).

[9] C. Brunet et al., "Design, fabrication and validation of an OAM fiber supporting 36 states", Optics Express, Vol. 22, n. 21, p. 26117 (2014).

[10] W. Xuli et al., "Orbital Angular Momentum Multiplexing in 0.1-THz Free-Space Communication via 3D Printed Spiral Phase Plates", Proc. CLEO, STu2F.2, San Jose (2014).

[11] M. J. Strain et al., "Fast electrical switching of orbital angular momentum modes using ultra-compact integrated vortex emitters", Nature Comm., Vol. 5, n. 4856, (2014).

[12] N. K. Fontaine et al., "Efficient multiplexing and demultiplexing of freespace orbital angular momentum using photonic integrated circuits", Proc. OFC, OTu1I.2, Los Angeles (2012).

[13] C. Quin et al., "Demonstration of Orbital Angular Momentum State Conversion using Two Hybrid 3D Photonic Integrated Circuits", Proc. OFC, Th4A.1 San Francisco (2014).

[14] http://www.juniper.net/us/en/local/pdf/datasheets/1000480-en.pdf

[15] "Lippis Report: Open Industry Network Performance \& Power Test for Cloud Networks Evaluating 10/40 GbE Switches", Fall 2013 Edition, December 2013.

[16] K. G. Brill, "The invisible crisis in the data center: the economic meltdown of Moore's law," White Paper, Uptime Institute, 2007.

[17] D. A. B. Miller, "Device requirements for optical interconnects to silicon chips,” Proc. IEEE, vol. 97, no. 7, pp. 1166-1185, July 2009.

[18] N. Andriolli, P. G. Raponi, P. Castoldi, A. Bogoni, L. Potì, A. Bianchi; "Synchronous packet switches," PCT/EP2009/059412, WO/2010/127716, filed on Jul. 22, 2009, published on Nov. 11, 2010.

[19] J. Wang, J.Y. Yang, I.M. Fazal, N. Ahmed, Y. Yan, H. Huang, Y. Ren, Y. Yue, S. Dolinar, M. Tur, and A. E. Willner, "Terabit free-space data transmission employing orbital angular momentum multiplexing," Nature Photon.,vol. 6, pp. 488-496, 2012.

[20] X Cai et al, "Integrated compact optical vortex beam emitters," Science, vol. 338 , pp. 363-366, 2012. 